\title{
Overexpression of the Pluripotent Stem Cell Marker Podocalyxin in Prostate Cancer
}

\author{
ELISABETH I. HEATH ${ }^{1}$, LANCE K. HEILBRUN ${ }^{1}$, DARYN SMITH ${ }^{1}$, WILLIAM M. SCHOPPERLE ${ }^{2}$, \\ YAWEN JU ${ }^{2}$, SUSAN BOLTON ${ }^{1}$, QURATULAIN AHMED ${ }^{3}$ and WAEL A. SAKR ${ }^{3}$ \\ ${ }^{1}$ Barbara Ann Karmanos Cancer Institute, Department of Oncology, \\ Wayne State University School of Medicine, Detroit, MI, U.S.A.; \\ ${ }^{2}$ CureMeta LLC, Boston, MA, U.S.A.; \\ ${ }^{3}$ Department of Pathology, Wayne State University School of Medicine, Detroit, MI, U.S.A.
}

\begin{abstract}
Background/Aim: Podocalyxin, a member of the CD34 family of cell surface sialomucins, is overexpressed in human embryonal carcinoma cell lines, as well as in several cancer types, and is associated with poor prognosis. Podocalyxin variants are associated with an increased risk and aggressiveness of prostate cancer. Herein podocalyxin protein expression in prostate cancer was characterized. Materials and Methods: Expression of podocalyxin as well as of TRA-1-60 and TRA-1-81 antigens was assessed immunohistochemically in 84 radical prostatectomy specimens and in adjacent normal tissues. Results: Podocalyxin expression and H-scores were considerably higher in prostate tumors compared to normal tissues. High TRA-1-60 and TRA-1-81 staining was detected, however, in a much smaller percentage of prostate tumors, while their expression and H-scores were low in normal tissues. Similar trends for all three proteins were observed in prostatic intraepithelial neoplasia. Conclusion: Overexpression of podocalyxin in prostate cancer renders the protein a putative immunohistochemical marker of prostate cancer that may contribute to stratification of patients for optimal treatment.
\end{abstract}

Podocalyxin, a member of the CD34 family of cell surface sialomucins, is expressed in normal cells (e.g., hematopoietic stem and progenitor cells, vascular epithelial cells and kidney podocytes), and regulates cellular morphology and adhesion $(1,2)$. Besides its role in health, podocalyxin has emerged as a predictor of poor prognosis and metastasis in

Correspondence to: Doctor Elisabeth I. Heath, Wayne State University Karmanos Cancer Institute, 4100 John R, HudsonWebber Cancer Research Center Bldg., Room 4234, Detroit, MI 48201, U.S.A. Tel: +1 3135768624, Fax: +1 3135768767, e-mail: heathe@karmanos.org

Key Words: Biomarkers, immunohistochemistry, pluripotent stem cells, podocalyxin expression, prostate cancer. several cancer types, including breast, esophageal, gastric, pancreatic, colorectal and urothelial bladder cancer (3-10). Of note, Casey et al. (11) showed that germline in-frame deletions in podocalyxin are associated with increased risk and aggressiveness of prostate cancer.

Schopperle et al. $(12,13)$ discovered that podocalyxin together with TRA-1-60 and TRA-1-81 antigens were overexpressed in human embryonal carcinoma cell lines, which are representative of malignant pluripotent stem cells. Toyoda et al. (14) identified podocalyxin as a major pluripotent marker of human embryonic stem cells and induced pluripotent stem cells. Because of their localization at the plasma membrane, podocalyxin, TRA-1-60 and TRA-1-81 can be used to detect cancer cells with stem cell properties in tumors. Cancer stem cells have been proposed to drive primary tumor aggressiveness, metastases and therapeutic resistance (15). Identifying cancer stem cells in the primary tumor may provide prognostic information that could affect treatment decisions.

A number of putative cancer stem cell markers have been identified in prostate cancer (15). Elevated gene expression of podocalyxin in metastatic prostate tumors, but not in primary prostate tumors was shown by microarray (cf. Gene Expression Omnibus, https://www.ncbi.nlm.nih.gov/geo, dataset GSE6919) (16-18). Siu et al. (19) showed that TRA-1-60 expression was associated with aggressive oral cancer invasion. Using prostate tumors, Rajasekhar et al. (20) purified stem-like tumor initiating cells co-expressing TRA-1-60 with CD151 and CD166, and showed that this subpopulation of cells was able to recapitulate the parent prostate tumor in mouse xenotransplantation models.

Based on the lack of correlation between podocalyxin mRNA levels and protein expression in colorectal cancer, Larsson et al. (4) argued that immunohistochemistry was the most suitable method for determining podocalyxin protein expression in the clinical setting. In prostate cancer, however, immunohistochemical evidence of podocalyxin protein 
expression is missing. Hence, this study aimed to determine immunohistochemical expression of podocalyxin, TRA-1-60 and TRA-1-81 in prostate tumors of various histopathological patterns (Gleason scores of 6, 7, and 8-9), and in adjacent normal tissues, and to assess their potential association with prostate cancer.

\section{Materials and Methods}

Patient samples. This was a retrospective, single-institution pilot study. The study was approved by the institutional review board. Prostate tumor and adjacent normal tissue specimens were obtained from each of the 84 consecutive patients who had undergone radical prostatectomy at the Karmanos Cancer Institute (Detroit, MI, USA), resulting in 168 samples for analysis.

Immunohistochemical staining and histological evaluation. Radical prostatectomy tissue samples were used to prepare 4- $\mu$ m formalinfixed, paraffin-embedded sections that were mounted on glass slides. The slides were deparaffinized, rehydrated, and incubated with the appropriate primary antibody at $4^{\circ} \mathrm{C}$ for $18 \mathrm{~h}$, followed by incubation with a biotin-conjugated secondary antibody for $1 \mathrm{~h}$ at room temperature. After incubation with peroxidase-conjugated streptavidin complex (Vectastain ABC Kit, Vector Laboratories, Burlingame, CA, USA) for $1 \mathrm{~h}$ at room temperature and three subsequent washes, the slides were developed using 3,3-diaminobenzidine tetrahydrochloride tablets (Dako, Glostrup, Denmark) for $5 \mathrm{~min}$. For podocalyxin detection, anti-podocalyxin-like 1 (3D3) mouse monoclonal antibody (1:500; Santa Cruz Biotechnology, Santa Cruz, CA, USA) and biotinconjugated anti-mouse IgG antibody (Sigma-Aldrich, St Louis, MO, USA) were used as the primary and secondary antibody, respectively. For TRA detection, anti-TRA-1-60 and anti-TRA-1-81 mouse monoclonal antibodies (1:1,000; EMD Millipore, Darmstadt, Germany) were used as primary antibodies and anti-mouse polyclonal IgM antibody (Sigma-Aldrich) was used as the secondary antibody. Slides were counterstained with hematoxylin and eosin, dehydrated, coverslipped and microscopically reviewed. Areas of low $(\leq 6)$ intermediate (7) and high-grade $(\geq 8)$ tumors, along with areas of highgrade prostatic intraepithelial neoplasia and benign prostatic epithelium were identified. The immunohistochemical expression of podocalyxin, TRA-1-60 and TRA-1-81 was assessed semi-quantitatively by two metrics: the extent of distribution and the intensity of staining. Based on the extent of distribution, the approximate percentage of cells with any positive immunohistochemical staining was estimated and the following scoring system was applied: 1 (0-24\% cells), 2 (25-49\% cells), 3 (50-74\% cells) and 4 ( $\geq 75 \%$ cells). The intensity of staining was graded as 1 (weak), 2 (moderate), 3 (strong) and 4 (very strong).

Statistical methods. Podocalyxin, TRA-1-60 and TRA-1-81 expression signatures in Gleason grade 6,7 , or $\geq 8$ tumors and in adjacent normal tissues were determined in a total of six groups. The percentage of patients that were scored $\geq 3$ for staining intensity corresponding to the expression level of each protein was estimated separately within each group. To estimate each percentage with a two-sided Wilson type confidence interval (CI) width of 0.30 at the $90 \%$ confidence level, regardless of the true value of the percentage, an $\mathrm{N}=28$ men per group was required. This sample size was determined using PASS 11 software (NCSS, LLC, Kaysville, UT, USA; htpps://www.ncss.com). Thus, across the three Gleason score
Table I. Clinical characteristics of patients.

\begin{tabular}{lcccc}
\hline Variable & \multicolumn{4}{c}{ Gleason score } \\
\cline { 2 - 5 } & 6 & 7 & $8-9$ & All \\
\hline Cases (N) & 28 & 28 & 28 & 84 \\
Age* (years; mean) & 56.8 & 60.1 & 60.9 & 59.3 \\
Age (years; range) & $47.5-66.5$ & $48.0-74.4$ & $45.3-78.8$ & $45.3-78.8$ \\
Race (N) & & & & \\
$\quad$ Caucasians & 13 & 14 & 0 & 27 \\
$\quad$ African Americans & 15 & 14 & 28 & 57 \\
Androgen deprivation & & & & \\
treatment (N) & 24 & 19 & 17 & 60 \\
$\quad$ No & 0 & 1 & 10 & $11^{\dagger}$ \\
$\quad$ Yes & 4 & 8 & 1 & 13 \\
$\quad$ Unknown & & & & \\
\hline
\end{tabular}

*Age of patients at radical prostatectomy; one patient's age could not be determined. Androgen deprivation treatment began after radical prostatectomy for all patients except one. N: Actual number of patients.

groups a total of 84 samples was required. The actual sample size for protein staining, however, was often less than 84 because of the absence of a pathology component on some of the slides. In addition to using percentages, bivariate relationships (i.e., for tumor tissue $v s$. adjacent normal tissue, or for one protein $v s$. another protein) were examined using two-way frequency distributions.

\section{Results}

Clinical characteristics of patients. Prostate cancer patients were categorized into three groups with Gleason scores 6, 7, and 8-9 (Table I). Age at radical prostatectomy was comparable in all three groups. The race distribution was comparable for men with Gleason scores 6 and 7. However, the Gleason score 8-9 prostate cancer samples were all from African American men. Following post-radical prostatectomy, 11 men (13\%) underwent androgen deprivation treatment, 22 (26\%) had biochemical recurrence, 8 (10\%) developed distant metastatic disease, and $6(7 \%)$ expired.

Immunohistochemical detection of podocalyxin, TRA-1-60 and TRA-1-81 in prostate cancer. The sensitivity of immunohistochemical staining intensity of each protein was assessed and the data are shown in Table II. Very high sensitivity of podocalyxin staining was detected in highgrade tumors (0.98). Similarly, high sensitivity of podocalyxin staining was observed in low-grade tumors $(0.88)$ and in prostatic intraepithelial neoplasia (0.81). In contrast, the sensitivity of TRA-1-60 and TRA-1-81 staining was extremely low in all three tumor types.

Staining intensities corresponding to the expression level of each protein in high-grade tumors are depicted in Figure 1. Podocalyxin staining was strong or very strong in high-grade 
Heath et al: Podocalyxin Overexpression in Prostate Cancer

Table II. Sensitivity of immunohistochemical staining intensity of podocalyxin, TRA-1-60 and TRA-1-81 in prostate cancer.

\begin{tabular}{lccc}
\hline Biomarker & High-grade tumors & Low-grade tumors & PIN \\
\hline Podocalyxin & $54 / 55=0.98(0.92-1.00)$ & $71 / 81=0.88(0.80-0.92)$ & $62 / 77=0.81(0.72-0.87)$ \\
TRA-1-60 & $8 / 55=0.15(0.08-0.24)$ & $17 / 80=0.21(0.15-0.30)$ & $8 / 74=0.11(0.06-0.18)$ \\
TRA-1-81 & $7 / 54=0.13(0.07-0.22)$ & $12 / 78=0.15(0.10-0.23)$ & $6 / 75=0.08(0.04-0.15)$ \\
\hline
\end{tabular}

Only prostate tumor tissue slides were used to assess the sensitivity of the staining intensity for each protein. Point estimates of the sensitivity of the staining intensity with the $90 \%$ Wilson type confidence limits for each protein are shown in parentheses. PIN: Prostatic intraepithelial neoplasia.

tumors on $56 \%$ and $20 \%$ of 55 slides, respectively, yielding a combined rate of $76 \%(90 \% \mathrm{CI}=66-84 \%)$. In contrast, staining of TRA-1-60 and TRA-1-81 was strong or very strong combined in this tumor type on only $13 \%(90 \% \mathrm{CI}=7-22 \%)$ of 55 slides and on $11 \%(90 \% \mathrm{CI}=6-20 \%)$ of 54 slides, respectively. Similarly, staining for the control biomarker IgG was strong or very strong in high-grade tumors on $11 \%$ and $2 \%$ of 55 slides, respectively, yielding a combined rate of $13 \%(90 \% \mathrm{CI}=7-22 \%)$. Furthermore, staining for the control biomarker IgM was not detectable above the expression level 2 (moderate staining) in this tumor type on any of 53 slides $(90 \% \mathrm{CI}=0-5 \%)$.

In addition, in low-grade tumors, podocalyxin staining was strong or very strong combined on $54 \%$ of 81 slides (90\% CI=45-63\%; data not shown). In the same tumor type, however, staining of TRA-1-60 and TRA-1-81 was strong or very strong combined on only $9 \%$ of 80 and 78 slides, respectively $(90 \% \mathrm{CI}=5-15 \%$; data not shown). Moreover, in prostatic intraepithelial neoplasia, podocalyxin staining was strong or very strong combined on $47 \%$ (90\% CI $=38-56 \%)$ of 77 slides. In contrast, in the same tumor type, staining of TRA-1-60 and TRA-1-81 was strong or very strong combined on only $7 \%(90 \% \mathrm{CI}=3-13 \%)$ and $3 \%(90 \% \mathrm{CI}=1-$ $8 \%$ ) of 74 and 75 slides, respectively (data not shown).

$H$-scores for immunohistochemical staining of podocalyxin, TRA-1-60 and TRA-1-81 in prostate cancer. H-scores assigned to each biomarker in high-grade tumors are shown in Figure 2. Podocalyxin staining had a high $\mathrm{H}$-score (median of 12.0). In contrast, staining of TRA-1-60 and TRA-1-81, as well as of the controls $\operatorname{IgG}$ and $\operatorname{IgM}$ were all assigned low H-scores (medians of 0.0 uniformly). In addition, in low-grade tumors, podocalyxin staining had a high $\mathrm{H}$-score (median of 9.0). In normal tissues, however, podocalyxin staining was assigned a low H-score (median of 3.0). Staining of TRA-1-60 and TRA1-81 was given low $\mathrm{H}$-scores (medians of 0.0 uniformly) in low-grade tumors, as well as in normal tissues. In addition, in prostatic intraepithelial neoplasia, median $\mathrm{H}$-scores for the staining of podocalyxin, TRA-1-60, and TRA-1-81 were 8.0, 0.0 , and 0.0 , respectively.

Additional immunohistochemical detection of podocalyxin in prostate cancer. As depicted in Figure $3(\mathrm{~A}-\mathrm{C})$, the presence of podocalyxin was detected by immunohistochemical staining in high-grade and low-grade prostate cancer, but not in adjacent benign epithelium. In high-grade prostate cancer (Gleason score $\geq 8$ ), both the extent of distribution and the intensity of staining of podocalyxin were assigned a score of 4 , i.e. $\geq 75 \%$ cells displayed very strong staining. In low-grade prostate cancer (Gleason score 6), the extent of distribution and the intensity of staining of podocalyxin were assigned scores of 4 and 3 , respectively, i.e. $\geq 75 \%$ of cells displayed strong staining. In contrast, in adjacent benign epithelium the intensity of staining and the extent of distribution were assigned scores of 1 and 3, respectively, i.e. weak staining was observed in $50-74 \%$ of cells. The data strongly support the notion that podocalyxin protein expression is greater in highgrade and low-grade prostate tumors than in normal tissues.

In addition, the relationship between the staining intensity of podocalyxin and the cellular distribution of staining in high-grade tumors is shown in Figure 4. Specifically, 23\% of 31 tumors $(90 \% \mathrm{CI}=13-37 \%)$ contained $50-74 \%$ cells with strong staining (score 3 ) for podocalyxin. Importantly, $74 \%$ of the same group of tumor samples $(90 \% \mathrm{CI}=60-85 \%)$ contained $75-100 \%$ cells with strong staining (score 3 ) for podocalyxin. In addition, $18 \%$ of 11 tumors $(90 \% \mathrm{CI}=6-43 \%)$ contained $50-74 \%$ cells with very strong staining (score 4 ) for podocalyxin. Moreover, $82 \%$ of the same group of tumor samples $(90 \% \mathrm{CI}=57-94 \%)$ contained $75-100 \%$ cells with very strong staining (score 4 ) for podocalyxin. The results suggest that the percentage of prostate cancer cells with high expression of podocalyxin is high in high-grade tumors.

\section{Discussion}

To our knowledge, this is the first report showing immunohistochemical evidence for high podocalyxin expression in prostate cancer. Of note, podocalyxin expression was considerably higher in prostate cancer and prostatic intraepithelial neoplasia compared to adjacent normal tissue. Interestingly, Schopperle et al. (12) reported in an earlier study, that there was no expression of podocalyxin in LnCap human prostate carcinoma cells. The apparent discrepancy between in vitro and human tissue data underscores the need for using clinical samples to validate cell culture data (21). 


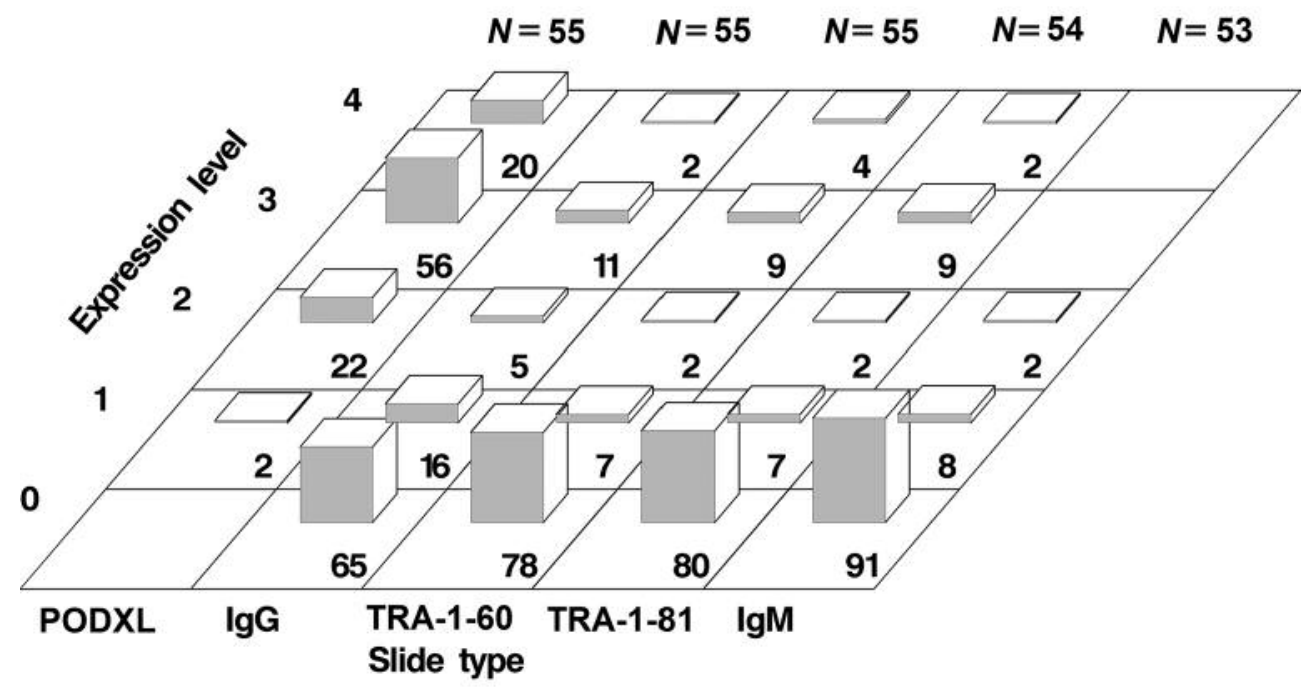

Figure 1. Immunohistochemical staining intensity of protein markers in high-grade tumors. The percentage of slides with tumor samples for a given staining intensity is indicated by the number at the bottom of each bar. Staining intensity corresponds to the expression level of each protein. Ig: Immunoglobulin; N: number of slides containing tumor specimens, each derived from a patient; PODXL: podocalyxin.

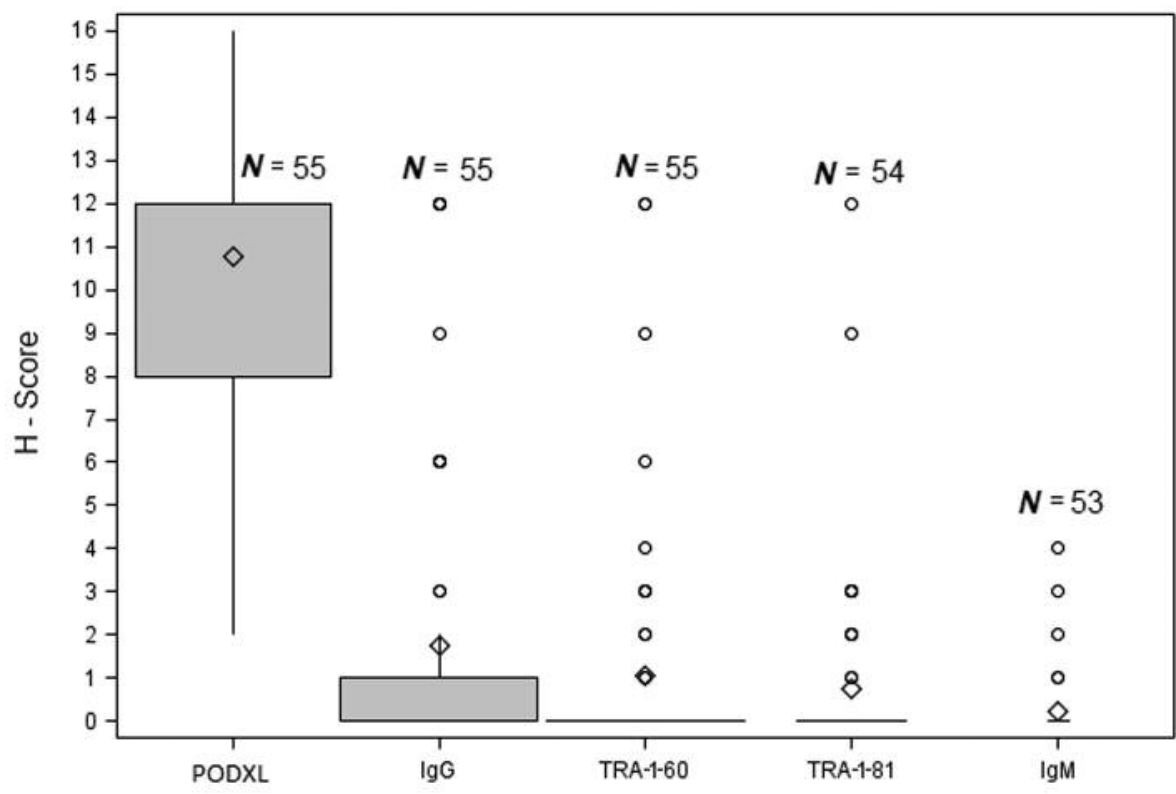

Figure 2. H-scores for immunohistochemical staining of protein markers in high-grade tumors. In the box plot, the diamond represents the mean, the upper and the lower bounds of the box represent the 75th and 25th percentiles, respectively, and the whiskers represent $90 \%$ confidence interval for the mean. Ig: Immunoglobulin; $N$ : number of slides containing tumor specimens, each derived from a patient; PODXL: podocalyxin.

In contrast to podocalyxin, high staining of both TRA-1-60 and TRA-1-81 was detect in a smaller percentage of prostate tumors. The data indicate that the expression level of both TRA-1-60 and TRA-1-81 was not associated with the tumor grade in prostate tumors. Notably, the percentage of highgrade tumors with high staining for both TRA-1-60 and TRA-1-81 correlates well with the predicted number of cases of localized prostate cancer that would eventually develop into metastatic disease. To designate TRA-1-60 and TRA-1-81 as a marker for metastatic disease would require, however, a larger study than the present report.

The mechanism of podocalyxin action has been studied by other groups. Specifically, Sizemore et al. (22) showed that podocalyxin overexpression resulted in up-regulation of 

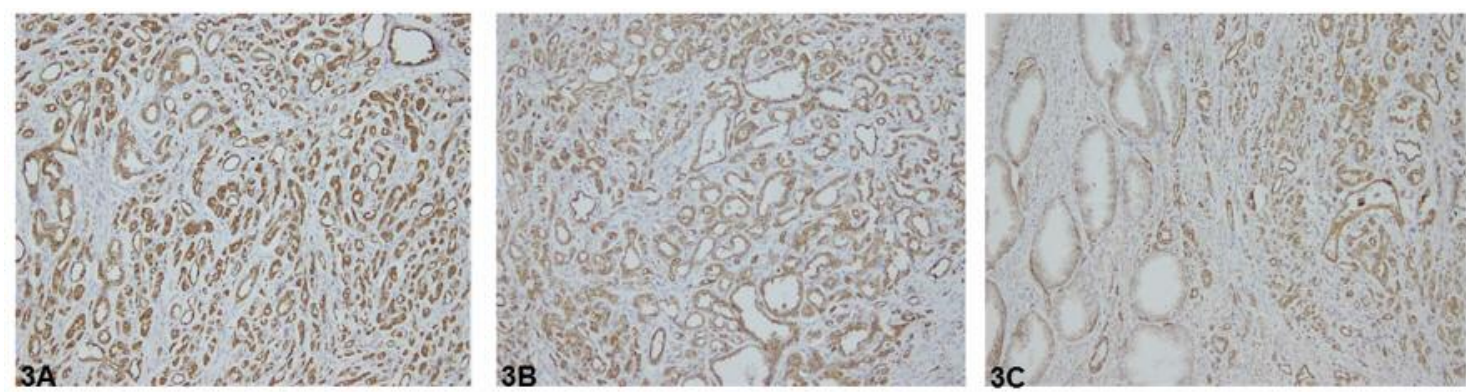

Figure 3. Podocalyxin immunohistochemistry in prostate cancer. A: High-grade prostate cancer. B: Low-grade prostate cancer. C: Benign epithelium (left) and low-grade prostate cancer (right). Original magnification $\times 400$.

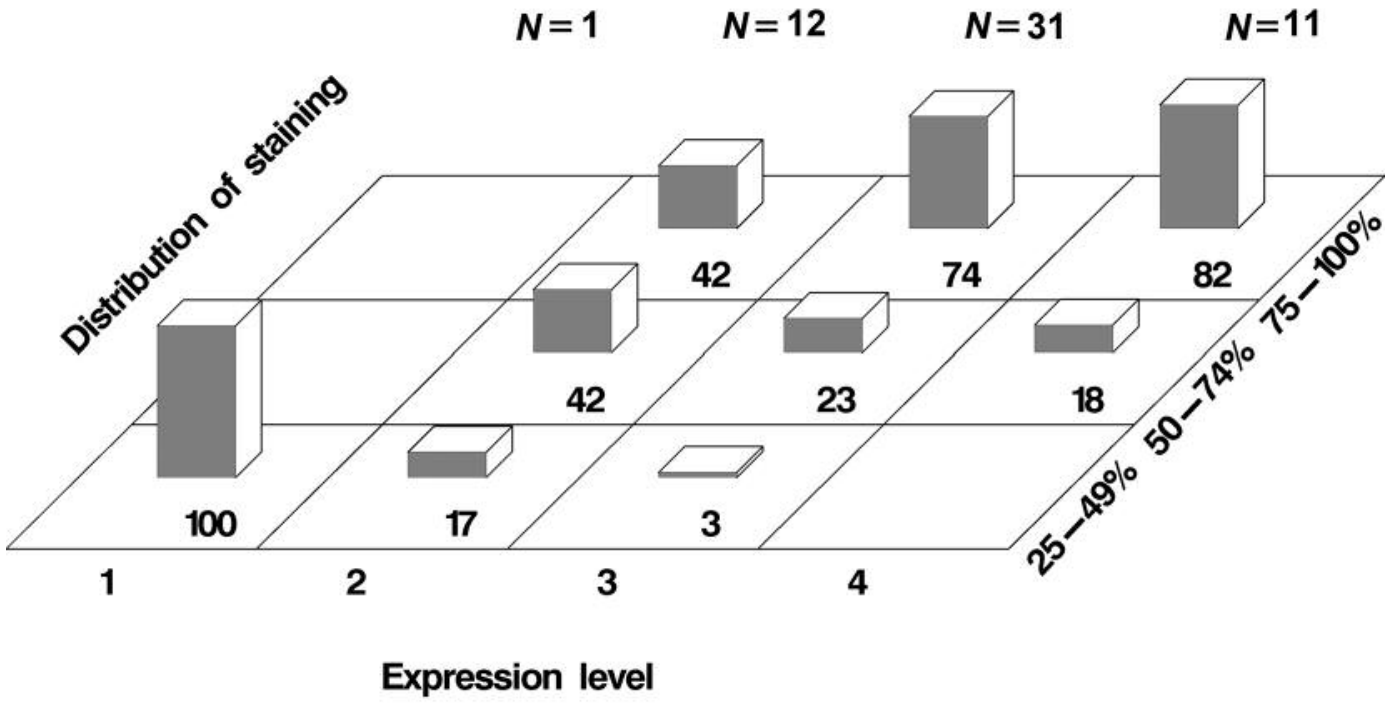

Figure 4. Cellular distribution of podocalyxin immunohistochemical staining in high-grade tumors. The percentage of slides with tumor samples for a range of cellular distribution of staining at a given staining intensity is indicated by the number at the bottom of each bar. Staining intensity corresponds to the expression level of podocalyxin. $N$ : Number of slides containing tumor specimens, each derived from a patient.

matrix metalloproteases and MAPK, activation of PI3K, as well as increased migration and invasion of prostate cancer cells. They also demonstrated that the in vitro aggressive phenotype was associated with the interaction of podocalyxin with metastasis-related ezrin. Most recently, Frose et al. (23) demonstrated that podocalyxin was induced by epithelialmesenchymal transition as a mediator of extravasation by direct interaction with ezrin, with subsequent metastasis formation. Furthermore, Snyder et al. (24) showed that podocalyxin was involved in breast cancer progression and validated podocalyxin as a tentative target for monoclonal antibody therapy to counteract primary tumor growth and systemic dissemination. Overall, the evidence discussed here strongly supports the idea that podocalyxin is a predictor of poor prognosis and distant metastasis, as well as a potential therapeutic target.

\section{Conclusion}

Our findings support the notion that podocalyxin is an immunohistochemical, cancer stem cell and prognostic marker of prostate cancer with the potential of contributing to (i) stratifying patients for optimal treatment, (ii) predicting biological progression, and (iii) guiding treatment decisions. Because of the dramatic decrease in survival of patients with metastatic prostate cancer compared to patients with localized disease, identifying tumors that are likely to recur and preventing metastases from occurring are of great interest. Moreover, a number of other putative cancer stem cell markers have been implicated in prostate cancer, including $\alpha 2$ - and $\alpha 6$-integrins (25), CD44 (26), CD166 (20, 27 ) and Trop2 (28), and we plan on exploring their role in prostate cancer progression in the future. 


\section{Acknowledgements}

This study was funded, in part, by the Cancer Center Support Grant P30 CA022453 from the National Institutes of Health.

\section{References}

1 Nielsen JS and McNagny KM: Novel functions of the CD34 family. J Cell Sci 121(Pt 22): 3683-3692, 2008.

2 Nielsen JS and McNagny KM: The role of podocalyxin in health and disease. J Am Soc Nephrol 20(8): 1669-1676, 2009.

3 Somasiri A, Nielsen JS, Makretsov N, McCoy ML, Prentice L, Gilks CB, Chia SK, Gelmon KA, Kershaw DB, Huntsman DG, McNagny KM and Roskelley CD: Overexpression of the antiadhesin podocalyxin is an independent predictor of breast cancer progression. Cancer Res 64(15): 5068-5073, 2004.

4 Larsson A, Fridberg M, Gaber A, Nodin B, Leveen P, Jonsson $\mathrm{G}$, Uhlen $\mathrm{M}$, Birgisson $\mathrm{H}$ and Jirstrom $\mathrm{K}$ : Validation of podocalyxin-like protein as a biomarker of poor prognosis in colorectal cancer. BMC Cancer 12: 282, 2012.

5 Boman K, Larsson AH, Segersten U, Kuteeva E, Johannesson H, Nodin B, Eberhard J, Uhlen M, Malmstrom PU and Jirstrom $\mathrm{K}$ : Membranous expression of podocalyxin-like protein is an independent factor of poor prognosis in urothelial bladder cancer. Br J Cancer 108(11): 2321-2328, 2013.

6 Forse CL, Yilmaz YE, Pinnaduwage D, O'Malley FP, Mulligan AM, Bull SB and Andrulis IL: Elevated expression of podocalyxin is associated with lymphatic invasion, basal-like phenotype, and clinical outcome in axillary lymph node-negative breast cancer. Breast Cancer Res Treat 137(3): 709-719, 2013.

7 Laitinen A, Bockelman C, Hagstrom J, Kokkola A, Fermer C, Nilsson $\mathrm{O}$ and Haglund $\mathrm{C}$ : Podocalyxin as a prognostic marker in gastric cancer. PLoS One 10(12): e0145079, 2015.

8 Saukkonen K, Hagstrom J, Mustonen H, Juuti A, Nordling S, Fermer C, Nilsson O, Seppanen $\mathrm{H}$ and Haglund C: Podocalyxin is a marker of poor prognosis in pancreatic ductal adenocarcinoma. PLoS One 10(6): e0129012, 2015.

9 Borg D, Hedner C, Nodin B, Larsson A, Johnsson A, Eberhard $\mathrm{J}$ and Jirstrom $\mathrm{K}$ : Expression of podocalyxin-like protein is an independent prognostic biomarker in resected esophageal and gastric adenocarcinoma. BMC Clin Pathol 16: 13, 2016.

10 Wang J, Zhao Y, Qi R, Zhu X, Huang C, Cheng S, Wang S and Qi X: Prognostic role of podocalyxin-like protein expression in various cancers: A systematic review and meta-analysis. Oncotarget 8(32): 52457-52464, 2017.

11 Casey G, Neville PJ, Liu X, Plummer SJ, Cicek MS, Krumroy LM, Curran AP, McGreevy MR, Catalona WJ, Klein EA and Witte JS: Podocalyxin variants and risk of prostate cancer and tumor aggressiveness. Hum Mol Genet 15(5): 735-741, 2006.

12 Schopperle WM, Kershaw DB and DeWolf WC: Human embryonal carcinoma tumor antigen, Gp200/GCTM-2, is podocalyxin. Biochem Biophys Res Commun 300(2): 285-290, 2003.

13 Schopperle WM and DeWolf WC: The TRA-1-60 and TRA-1-81 human pluripotent stem cell markers are expressed on podocalyxin in embryonal carcinoma. Stem Cells 25(3): 723-730, 2007.

14 Toyoda H, Nagai Y, Kojima A and Kinoshita-Toyoda A: Podocalyxin as a major pluripotent marker and novel keratan sulfate proteoglycan in human embryonic and induced pluripotent stem cells. Glycoconj J 34(2): 139-145, 2017.

15 Harris KS and Kerr BA: Prostate cancer stem cell markers drive progression, therapeutic resistance, and bone metastasis. Stem Cells Int 2017: 8629234, 2017.
16 Yu YP, Landsittel D, Jing L, Nelson J, Ren B, Liu L, McDonald C, Thomas R, Dhir R, Finkelstein S, Michalopoulos G, Becich $\mathrm{M}$ and Luo JH: Gene expression alterations in prostate cancer predicting tumor aggression and preceding development of malignancy. J Clin Oncol 22(14): 2790-2799, 2004.

17 Chandran UR, Ma C, Dhir R, Bisceglia M, Lyons-Weiler M, Liang W, Michalopoulos G, Becich M and Monzon FA: Gene expression profiles of prostate cancer reveal involvement of multiple molecular pathways in the metastatic process. BMC Cancer 7: 64, 2007.

18 McNagny K, Hughes M, Graves M, Debruin E, Snyder K, Cipollone J, Turvey M, Tan P, McColl S and Roskelley DC: Podocalyxin in the diagnosis and treatment of cancer. In: Advances in Cancer Management (Mohan R, ed.). IntechOpen, 2012.

19 Siu A, Lee C, Dang D, Lee C and Ramos DM: Stem cell markers as predictors of oral cancer invasion. Anticancer Res 32(4): 1163-1166, 2012.

20 Rajasekhar VK, Studer L, Gerald W, Socci ND and Scher HI: Tumour-initiating stem-like cells in human prostate cancer exhibit increased NF-kappaB signalling. Nat Commun 2: 162, 2011.

$21 \mathrm{Niu} \mathrm{N}$ and Wang L: In vitro human cell line models to predict clinical response to anticancer drugs. Pharmacogenomics 16(3): 273-285, 2015.

22 Sizemore S, Cicek M, Sizemore N, Ng KP and Casey G: Podocalyxin increases the aggressive phenotype of breast and prostate cancer cells in vitro through its interaction with ezrin. Cancer Res 67(13): 6183-6191, 2007.

23 Frose J, Chen MB, Hebron KE, Reinhardt F, Hajal C, Zijlstra A, Kamm RD and Weinberg RA: Epithelial-mesenchymal transition induces podocalyxin to promote extravasation via ezrin signaling. Cell Rep 24(4): 962-972, 2018.

24 Snyder KA, Hughes MR, Hedberg B, Brandon J, Hernaez DC, Bergqvist P, Cruz F, Po K, Graves ML, Turvey ME, Nielsen JS, Wilkins JA, McColl SR, Babcook JS, Roskelley CD and McNagny KM: Podocalyxin enhances breast tumor growth and metastasis and is a target for monoclonal antibody therapy. Breast Cancer Res 17: 46, 2015.

25 Hoogland AM, Verhoef EI, Roobol MJ, Schroder FH, Wildhagen MF, van der Kwast TH, Jenster $G$ and van Leenders GJ: Validation of stem cell markers in clinical prostate cancer: alpha6-integrin is predictive for non-aggressive disease. Prostate 74(5): 488-496, 2014.

26 Chen X, Nagai Y, Zhu Z, Ruan H, Peehl DM, Greene MI and Zhang H: A spliced form of CD44 expresses the unique glycan that is recognized by the prostate cancer specific antibody F77. Oncotarget 9(3): 3631-3640, 2018.

27 Jiao J, Hindoyan A, Wang S, Tran LM, Goldstein AS, Lawson D, Chen D, Li Y, Guo C, Zhang B, Fazli L, Gleave M, Witte $\mathrm{ON}$, Garraway IP and Wu H: Identification of CD166 as a surface marker for enriching prostate stem/progenitor and cancer initiating cells. PLoS One 7(8): e42564, 2012.

28 Xie J, Molck C, Paquet-Fifield S, Butler L, Australian Prostate Cancer B, Sloan E, Ventura S and Hollande F: High expression of TROP2 characterizes different cell subpopulations in androgen-sensitive and androgen-independent prostate cancer cells. Oncotarget 7(28): 44492-44504, 2016.

Received September 22, 2018 Revised October 1, 2018 Accepted October 2, 2018 